\title{
Mortalidade por Câncer de Mama e Condições de Desenvolvimento Humano no Brasil
}

https://doi.org/10.32635/2176-9745.RBC.2019v65n1.50

\author{
Mortality for Breast Cancer and the Conditions of Human Development in Brazil \\ Mortalidad por Cáncer de Mama y Condiciones de Desarrollo Humano en Brasil
}

\author{
Larissa Di Leo Nogueira Costa'; Ana Hélia de Lima Sardinha²; Pabline Medeiros Verzaro³; Luciana Léda Carvalho Lisbôa4; \\ Rosangela Fernandes Lucena Batista ${ }^{5}$
}

Resumo

Introduçáo: $\mathrm{O}$ câncer de mama é considerado de relativo bom prognóstico se diagnosticado e tratado precocemente, com sobrevida média de 80\% após cinco anos do diagnóstico, porém o que se observa é que se trata da segunda neoplasia mais incidente em todo o mundo. Objetivo: Analisar o crescimento da taxa de mortalidade por câncer de mama e as condiçóes de desenvolvimento humano no Brasil. Método: Trata-se de um estudo descritivo de séries temporais nos dois períodos compreendidos entre 1998 a 2002 e 2008 a 2012. Resultados: Ao se analisarem as taxas de mortalidade e os índices de Desenvolvimento Humano (IDH), comparando o primeiro quinquênio (período de 1998-2002) com o segundo (período de 2008-2012), foi observado um aumento na taxa de mortalidade da maioria das capitais, com exceção de Maceió, Porto Alegre, Florianópolis e Palmas. Ocorreu um aumento do IDH em todas as capitais do país. Conclusáo: Houve crescimento nos índices de câncer de mama no Brasil de forma geral mesmo havendo também uma melhoria das condiçôes de desenvolvimento humano em todo o país. Palavras-chave: Neoplasias da Mama/mortalidade; Condiçóes Sociais; Indicadores de Desenvolvimento.

\begin{abstract}
Introduction: Breast cancer is disease of relatively good prognostics if diagnosed and treated precociously, an average of $80 \%$ of the patients survive after five years of the diagnoses, however what we observe is that it is the second neoplasm in the world. Objective: To analyze the growth of breast cancer mortality rate and the conditions of human development in Brazil. Method: It is a descriptive study of temporary series along the two terms from 1998 to 2002 and 2008 to 2012. Results: When we analyze the mortality rates and the Human Development Index, comparing the first five years (from 1998 to 2002) with the second term of five years (2008 to 2012), we observed a growth in the mortality rate in the majority of the capitals, except from Maceió, Porto Alegre, Florianópolis and Palmas. There was a growth in the HDI in all capitals of the country. There was a growth in the Human Development Index in all capitals of the country. Conclusion: There was a general growth in the breast cancer rates in Brasil, although the conditions of Human Development nation wide.

Key words: Breast Neoplasms/mortality; Social Conditions; Development Indicators.
\end{abstract}

Resumen

Introducción: El cáncer de mama es considerado de relativo buen pronóstico si se diagnostica y tratado precozmente con sobrevida media del $80 \%$ después de cinco años del diagnóstico, pero lo que se observa es que se trata de la segunda neoplasia más incidente en todo el mundo. Objetivo: Analizar el crecimiento de la tasa de mortalidad por cáncer de mama y las condiciones de desarrollo humano en Brasil. Método: Se trata de un estudio descriptivo de series temporales en los dos períodos comprendidos entre 1998 a 2002 y 2008 a 2012. Resultados: Ao se analizaren las tasas de mortalidad y los IDH comparando el primer quinquenio (período 19982002) con el segundo, (Período 2008-2012), se observó un aumento en la tasa de mortalidad de la mayoría de las capitales con excepción de Maceió, Porto Alegre, Florianópolis y Palmas. Y hubo un aumento del IDH en todas las capitales del país. Conclusión: Se ha producido un crecimiento del cáncer de mama en Brasil de forma general, incluso habiendo también una mejora de las condiciones de desarrollo humano en todo el país.

Palabras clave: Neoplasias de la Mama/mortalidad; Condiciones Sociales; Indicadores de Desarrollo.

\footnotetext{
${ }^{1}$ Universidade Federal do Maranhão (UFMA). São Luís (MA), Brasil. Orcid Id: https://orcid.org/0000-0003-3206-612X

2 UFMA. São Luís (MA), Brasil. Orcid iD: https://orcid.org/0000-0002-8720-6348

${ }^{3}$ UFMA. São Luís (MA), Brasil. Orcid iD: https://orcid.org/0000-0003-1449-1990

${ }^{4}$ UFMA. São Luís (MA), Brasil. Orcid iD: https://orcid.org/0000-0001-5370-3525

${ }^{5}$ UFMA. São Luís (MA), Brasil. Orcid iD: https://orcid.org/0000-0002-1529-0165

Endereço para correspondência: Larissa Di Leo Nogueira Costa. Universidade Federal do Maranhão. Avenida dos Portugueses, 1966 - Vila Bacanga. São Luís (MA), Brasil. CEP 65065-545. E-mail: larissadileonc@hotmail.com
} 


\section{INTRODUÇÃO}

O câncer de mama é uma das neoplasias mais temidas pelo gênero feminino em função do seu grande impacto psicológico, funcional e social, e pela sua influência negativa nas questóes relacionadas à autoimagem e à percepção da sexualidade. É o tipo de câncer mais comum entre as mulheres em todo o mundo, representando quase $25 \%$ de todos os casos de câncer ${ }^{1-3}$.

No Brasil, em 2018, quase 60 mil mulheres desenvolveram essa doença, segundo as estimativas do Instituto Nacional de Câncer José Alencar Gomes da Silva (INCA). Com exceção dos tumores de pele não melanoma, o câncer de mama é o mais frequente nas mulheres das Regióes Sudeste, Sul, Centro-Oeste e Nordeste, o que mostra sua relaçáo com estilos de vida e ambientes mais urbanizados. As taxas de mortalidade mais elevadas são observadas na Região Sul e nos Estados do Rio de Janeiro, de São Paulo e de Pernambuco ${ }^{3}$.

Embora a incidência em países de alta renda seja maior, sua mortalidade é menor e sua taxa vem sofrendo redução, diferentemente dos países em desenvolvimento, que possuem taxas de incidência e mortalidade crescentes ${ }^{4,5}$. Em países de média e baixa rendas, onde não existem programas eficientes para rastreamento dos cânceres de mama, os diagnósticos são realizados no momento em que já não existe mais possibilidade terapêutica para cura, a doença se encontra em estádio bastante avançado e nem o tratamento paliativo é capaz de diminuir o impacto da redução da sobrevida e o prognóstico de mortalidade ${ }^{4}$.

Os fatores sociais ligados à mortalidade por câncer de mama são pobreza, baixa escolaridade, residência na zona rural e, principalmente, falta de acesso aos serviços de saúde. A mortalidade é atenuada na proporção em que se inserem melhorias de acesso a medidas diagnósticas e terapêuticas ${ }^{5,6}$.

No Brasil, tendências de aumento da mortalidade por câncer de mama são verificadas de forma gradativa em todo o período de 1980-2012, nas cinco Regióes do país. Em algumas capitais, percebe-se uma tendência de declínio dos coeficientes de mortalidade a partir do final da década de 1990. O motivo desse declínio pode ser atribuído às maiores chances de diagnóstico das mulheres residentes em algumas capitais ${ }^{4,7}$.

Dessa forma, os fatores socioeconômicos são apontados como determinantes importantes na incidência e mortalidade por câncer, sendo reconhecidos como condicionantes de desigualdades na carga de câncer. As evidências demonstram que os grupos de níveis socioeconômicos mais baixos têm apresentado elevada mortalidade em razão de uma maior proporção de diagnósticos tardios, maior dificuldade de acesso ao diagnóstico e tratamento adequado, pior prognóstico, menor sobrevida e maior risco de óbito por câncer em geral e por tipos de cânceres potencialmente curáveis ${ }^{6}$.

Objetiva-se, por meio do presente estudo, analisar o crescimento da taxa de mortalidade por câncer de mama e as condiçóes de desenvolvimento humano nas capitais do Brasil nos quinquênios de 1998 a 2002 e de 2008 a 2012.

\section{MÉTODO}

Trata-se de uma pesquisa do tipo descritiva, retrospectiva, de séries temporais. A coleta de dados foi de forma secundária, utilizando as declaraçóes de óbito de todas as mulheres que possuíam a Classificação Internacional de Doenças e Problemas Relacionados à Saúde (CID-10) com código C50 (neoplasia maligna da mama) e suas variáveis (C50-C50.9), registradas em dois períodos de cinco anos (quinquênios), o primeiro período ou quinquênio, que inclui os anos de 1998 a 2002, e o segundo, que compreende o período de 2008 a 2012, em todas as capitais do Brasil e Distrito Federal. A escolha desses períodos foi realizada para que fossem englobados os anos em que foi realizada a pesquisa nacional do Índice de Desenvolvimento Humano (IDH), referente aos anos de 2000 e 2010, compreendendo dois anos antes e dois anos depois de cada pesquisa.

A coleta dos dados referentes ao IDH foi realizada por meio do Atlas de Desenvolvimento Humano do Brasil, disponibilizado pelo Instituto de Pesquisa Econômica Aplicada (IPEA) e pelo Instituto Brasileiro de Geografia e Estatística (IBGE) referentes às capitais ${ }^{8,9}$.

Para o cálculo da taxa de mortalidade, utilizou-se o método direto, aplicando a fórmula do cálculo da mortalidade por causa específica com o número de óbitos por câncer de mama em mulheres sobre o número de óbitos em mulheres, por 100 .

As taxas de mortalidade foram calculadas segundo faixa etária, raça/cor, escolaridade e capitais do Brasil. As taxas referentes ao primeiro quinquênio (1998 a 2002) foram comparadas com o IDH do ano de 2000 e as taxas referentes ao segundo quinquênio (2008 a 2012) foram comparadas com o IDH do ano de 2010.

Adotou-se, para as capitais, a classificação do IDH em três categorias: IDH baixo (entre 0 e 0,49 ), IDH médio (entre 0,5 e 0,79 ) e IDH alto (entre 0,8 e 1 ) dos anos de 2000 e $2010^{10}$.

Os dados foram analisados pelo programa Stata versão 12 (StataCorp., College Station, Estados Unidos da América), organizados e tabelados.

Foram empregados exclusivamente dados de acesso público, sem a identificação dos nomes nas declaraçôes de óbitos, atendendo às diretrizes éticas para a realização de pesquisas, e também, dessa forma, aos aspectos 
recomendados na resolução $466 / 12$, sobre pesquisa envolvendo seres humanos, respeitando-se os princípios éticos, a confidencialidade e o anonimato.

\section{RESULTADOS}

A mortalidade por câncer de mama cresceu de 41,38 do primeiro quinquênio (1998 a 2002) para 58,62 no segundo (2008 a 2012). As mulheres de cor branca representaram $71,13 \%$ no primeiro quinquênio e $61,47 \%$ no segundo. Mulheres pardas representaram 20,32\% e $28,43 \%$, seguido das mulheres pretas com $6,9 \%$ e 9,21 . A escolaridade mais frequente foi de quatro a sete anos de estudo formal, representando $26,22 \%$ a $26,46 \%$. A faixa etária predominante entre os óbitos por câncer de mama foi de mulheres com idade igual ou superior a 70 anos, dado observado em ambos os quinquênios, com 46,21\% e $50,29 \%$, seguido de mulheres com 50 a 59 anos, que representou $24,38 \%$ e $24,99 \%$ (Tabela 1 ).

Houve crescimento da taxa de mortalidade por câncer de mama em 23 capitais do país nos dois quinquênios. As cidades que apresentaram maior crescimento foram Boa Vista (84,21\%), Rio Branco (70,67\%) e Porto Velho $(58,59 \%)$ (Figura 1).

$\mathrm{Na}$ análise dos quinquênios separadamente, observou-se que Brasília $(2,88)$, Rio de Janeiro $(2,05)$ e São Paulo $(1,32)$ se mantiveram entre as cidades com as maiores taxas de mortalidade por câncer de mama de 1998 a 2002. As menores taxas observadas foram para Palmas $(0,46)$, Boa Vista $(0,41)$ e Rio Branco, São Luís e Florianópolis $(0,59)$.
Tabela 1. Dados sociodemográficos de óbito por câncer de mama nas capitais do Brasil. Brasil, 1998 a 2002 e 2008 a 2012

\begin{tabular}{lcccc}
\hline \multirow{2}{*}{ Variáveis } & \multicolumn{2}{c}{$\mathbf{1 9 9 8 - 2 0 0 2}$} & \multicolumn{2}{c}{$\mathbf{2 0 0 8 - 2 0 1 2}$} \\
\cline { 2 - 5 } Raça/Cor & $\mathbf{n}$ & $\%$ & $\mathbf{n}$ & $\%$ \\
Amarela & 272 & 1,56 & 215 & 0,81 \\
Branca & 12.387 & 71,13 & 16.377 & 61,47 \\
Indígena & 15 & 0,09 & 22 & 0,08 \\
Parda & 3.539 & 20,32 & 7.575 & 28,43 \\
Preta & 1.202 & 6,90 & 2.455 & 9,21 \\
Total & 17.415 & 100,00 & 26.644 & 100,00 \\
Escolaridade & & & & \\
$\geq 12$ anos & 2.561 & 17,77 & 3.794 & 18,20 \\
8 a 11 anos & 3.394 & 23,55 & 5.191 & 24,91 \\
4 a 7 anos & 3.778 & 26,22 & 5.515 & 26,46 \\
1 a 3 anos & 3.216 & 22,32 & 4.274 & 20,51 \\
Nenhum & 1.461 & 10,14 & 2.067 & 9,92 \\
Total & 14.410 & 100,00 & 20.841 & 100,00 \\
Idade & & & & \\
Até 19 anos & 3 & 0,02 & 4 & 0,01 \\
20 a 39 anos & 1.750 & 8,85 & 2.059 & 7,35 \\
40 a 49 anos & 4.033 & 20,40 & 4.799 & 17,12 \\
50 a 59 & 4.819 & 24,38 & 7.005 & 24,99 \\
60 a 69 & 29 & 0,15 & 67 & 0,24 \\
$\geq 70$ & 9.135 & 46,21 & 14.098 & 50,29 \\
Total & 19.769 & 100,00 & 28.032 & 100,00 \\
\hline
\end{tabular}

No segundo quinquênio, que compreende o período de 2008 a 2012, as capitais que apresentaram maior taxa de mortalidade por câncer de mama foram Brasília $(3,37)$, Sáo Paulo (3,01), Rio de Janeiro $(2,27)$ e Boa Vista $(1,93)$.

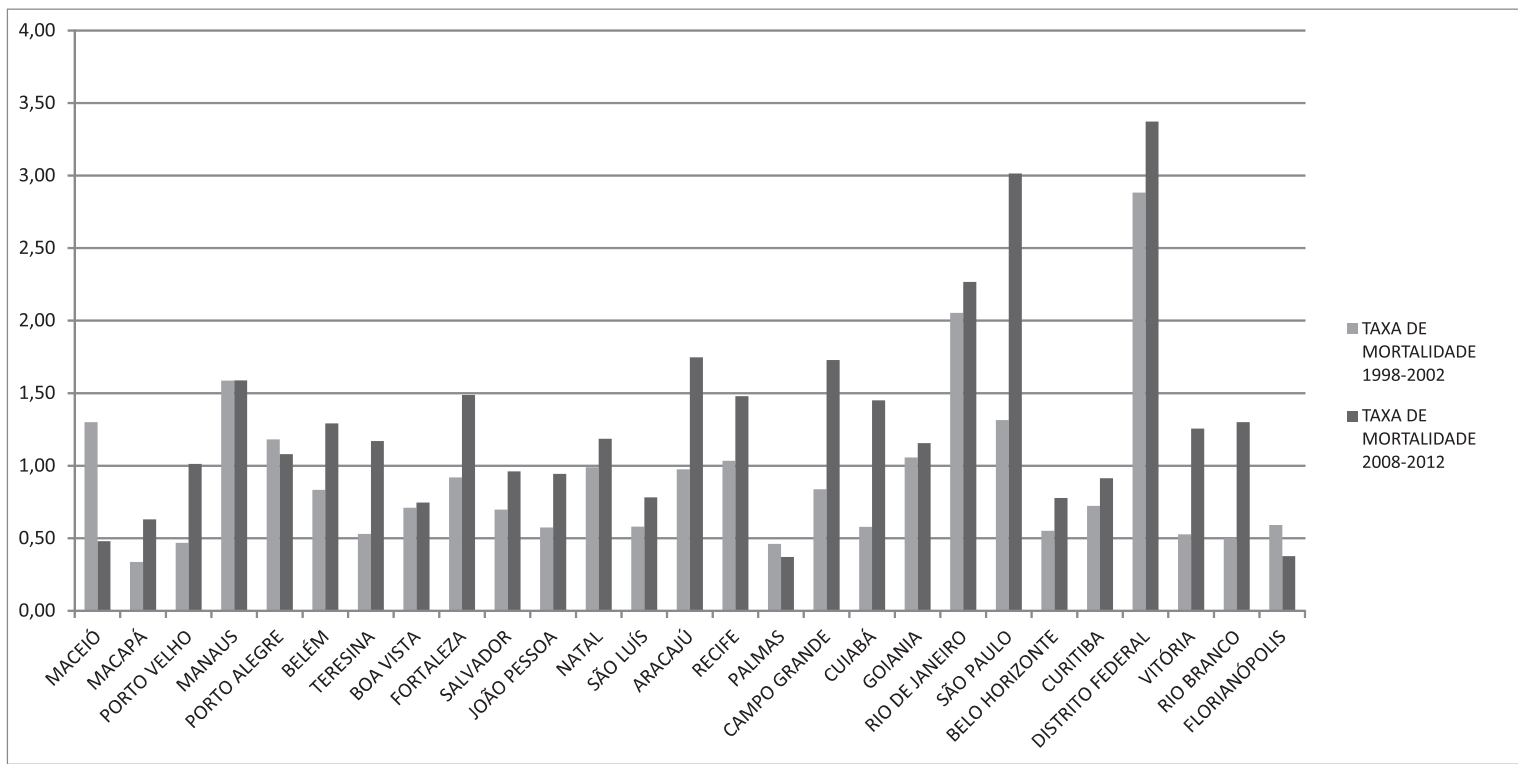

Figura 1. Taxa de mortalidade por câncer de mama. Brasil, 1998 a 2002 e 2008 a 2012 
As menores taxas foram de Maceió $(0,13)$, Florianópolis $(0,36)$ e Palmas $(0,37)$.

Com relação à análise do $\mathrm{IDH}$, as cidades que apresentaram melhor índice no ano de 2000 foram Florianópolis (0,76), Vitória e Curitiba com 0,75 e São Paulo com 0,73 . No ano de 2010, os melhores índices foram para Florianópolis e Vitória com 0,84, Brasília $(0,82)$, Curitiba $(0,76)$ e Belo Horizonte $(0,57)$. Os menores índices em 2000 e em 2010 foram para as mesmas cidades: Maceió, Rio Branco, Manaus e Porto Velho.

Dessa forma, ao se analisarem as taxas de mortalidade e os IDH, comparando o primeiro quinquênio (período de 1998-2002) com o segundo (período de 2008-2012), foi observado um aumento na taxa de mortalidade da maioria das capitais com exceção de Maceió, Porto Alegre, Florianópolis e Palmas. E com relação ao IDH, houve um aumento em todas as capitais do país.

\section{DISCUSSÃO}

A taxa de mortalidade foi analisada de acordo os indicadores sociodemográficos (raça/cor, escolaridade e idade) que demonstraram significância estatística entre um período (1998-2002) e outro (2008-2012) de $\mathrm{p}<0,001$.

No presente estudo, foi identificada uma maior mortalidade nas mulheres da cor branca, havendo um pequeno decréscimo, entre os períodos de 1998-2002 e de 2008-2012, de 71,13\% para 61,47\%. Nos estudos de Soares et al. ${ }^{10}$, sobre a mortalidade por câncer de mama feminino de acordo com a cor, também foi observada uma maior frequência na populaçáo feminina da cor branca. Além disso, houve um crescimento dessa mortalidade em todas as raças/ cores no período estudado pelo autor. Segundo o IBGE ${ }^{9}$, a população brasileira possui um alto grau de miscigenação entre as raças, apresentando $48,2 \%$ de raça/cor branca e $51,1 \%$ parda e preta, o que pode ser um dos motivos da distribuição de raça/cor encontradas neste estudo.

Com relação à faixa etária, a idade predominante de óbito, nos dois períodos estudados, é de mulheres com idade igual ou superior a 70 anos $(46,21 \%$ e $50,29 \%)$, seguido de mulheres de 50 a 59 anos (24,38\% e 24,99\%). Meira et al. ${ }^{11}$ realizaram um estudo sobre análise do efeito idade-período-corte na mortalidade por câncer de mama no Brasil e corroboraram este estudo ao constatarem que, em todas as Regióes geográficas, as taxas de mortalidade por câncer de mama aumentaram consideravelmente a partir da faixa etária de 50 a 54 anos, sendo verificadas maiores taxas nas mulheres com 75 anos e mais. Esse mesmo estudo concluiu ainda que houve um aumento na faixa etária de 75 anos e mais, no período de 1990 a 1994, persistindo até o período de 2000 a 2004.

É reconhecido que o rastreamento mamográfico após os 50 anos permite uma redução da mortalidade em mais de $30 \%$, tendo uma relação custo-benefício mais favorável quando comparado ao rastreamento aplicado em outras idades. Porém, os programas de rastreamento no Brasil iniciaram muito tardiamente e com coberturas bastante distintas entre as Regiôes ${ }^{11}$. No período de 1995 e 2002, o percentual de realização de mamografia pelo Sistema Único de Saúde (SUS) variou entre 17\% e 54\% do total de mulheres que deveriam realizar o exame, o que fica muito abaixo da média preconizada pelo Ministério da Saúde de $70 \%$ de cobertura ${ }^{12}$. Em 2008, houve uma cobertura de $71,5 \%$, representando as mulheres que obtiveram acesso ao exame uma vez na vida. O que explica, em parte, as altas taxas de mortalidade na faixa etária acima dos 60 anos encontradas em ambos os estudos ${ }^{12}$.

Dessa forma, parece evidente o efeito da idade, uma vez que a ela continua sendo um dos fatores de risco mais importantes para as taxas tanto de incidência quanto de mortalidade, fazendo com que tais discussóes sejam extremamente importantes para definição de políticas públicas adequadas ${ }^{10,11}$.

Em relação à taxa de mortalidade, houve um aumento na maioria das cidades estudadas, com diferenças maiores em Boa Vista, Rio Branco e Porto Velho. As únicas cidades que conseguiram diminuição das suas taxas foram Maceió, Palmas, Porto Alegre e Florianópolis. Um estudo realizado por Couto ${ }^{13}$ utilizou o período compreendido entre 1987 e 2013 para analisar a taxa de mortalidade por câncer de mama no Brasil e fatores associados. Nos seus estudos, também houve um aumento na taxa de mortalidade no Brasil de forma geral. A Região Sudeste foi a única a reduzir os índices de mortalidade em todo o período. As Regiōes Centro-Oeste, Norte e Nordeste, apresentaram crescimento em todo o período. E, na Região Sul, houve aumento entre 1990 e 2000, e diminuição entre 2000 e 2010.

$\mathrm{Na}$ presente pesquisa, as três cidades com maior crescimento na taxa de mortalidade (Boa Vista, Rio Branco e Porto Velho) se situam nas Regióes Norte e Centro-Oeste do país, que foram aquelas com crescimento de mortalidade segundo os estudos de Couto $^{13}$. Este estudo corrobora os dados desse autor, ao identificar a Região Sul como a de decréscimo da mortalidade, representada nesta pesquisa pelas capitais Porto Alegre e Florianópolis, que obtiveram uma diminuição significativa na taxa de mortalidade quando comparadas a outras capitais. Porém, este estudo demonstrou ainda decréscimo da mortalidade por câncer de mama nas cidades de Maceió e Palmas, que estão situadas na Regiáo Nordeste, contrapondo-se ao autor que indicou crescimento da mortalidade nessa Regiáo.

Em um estudo realizado por Barbosa et al. ${ }^{1}$ e, em um outro realizado por Girianelli et al. ${ }^{4}$, também foi identificado aumento em todos os Estados da Região Nordeste. Porém, Girianelli et al. ${ }^{4}$ observaram ainda um comportamento oposto quando analisadas somente as capitais, com 
decréscimo da mortalidade, corroborando o presente estudo. De acordo com os autores, essa reversão da mortalidade foi possível pelo acesso aos meios diagnósticos e terapêuticos, tornando-os efetivos nos estádios iniciais da doença.

Ao se analisarem as taxas de mortalidade e os IDH comparando os diferentes quinquênios, foi observado um aumento na taxa de mortalidade da maioria das capitais, com exceção de Maceió, Porto Alegre, Florianópolis e Palmas. E houve um aumento do IDH em todas as capitais do país.

No ano de 2000, os maiores IDH foram em Florianópolis $(0,76)$, Vitória e Curitiba com 0,75. E, no ano de 2010, foram para Florianópolis e Vitória com 0,84 e Brasília com 0,82 . Essas cidades, com exceçáo de Brasília, foram consideradas capitais com taxa de mortalidade baixa.

Segundo a pesquisa do Sistema de Vigilância de Fatores de Risco e Proteção para Doenças Crônicas por Inquérito Telefônico (Vigitel) ${ }^{12}$, realizada pelo Ministério da Saúde, Florianópolis está entre as cidades que apresentaram maior frequência de mulheres entre 50 a 69 anos de idade, que referiram ter realizado exame de mamografia nos últimos dois anos $(86,3 \%)$. O que pode significar, além de uma alta adesão das mulheres para realização do exame, reflexos da melhoria no acesso e condições de saúde das Regiōes ${ }^{14}$.

Em todas as capitais com os menores IDH, houve crescimento da taxa de mortalidade, com exceção de Porto Velho e Maceió. Porto Velho está inserido em uma Região de alta incidência de câncer de mama (Norte) e está entre as quatro cidades com menor IDH, porém, possui uma cobertura de mulheres com idade entre os 50 e 69 anos que realizam o exame de mamografia de $81,8 \% 0^{12,15}$. Maceió é uma das capitais onde a taxa de mortalidade mais reduziu do primeiro para o segundo quinquênio (de 0,6 para 0,13 ). A cidade possui $72,9 \%$ das mulheres entre 50 e 69 anos que realizaram a mamografia uma vez na vida ou realizaram nos últimos dois anos ${ }^{12}$. Isso pode nos indicar que as políticas direcionadas para a prevenção e o rastreamento do câncer de mama estão surtindo efeito, mesmo que o serviço ainda não possa ser visto como plenamente organizado ${ }^{15}$.

No estudo realizado por Couto ${ }^{13}$, não houve correlação entre o IDH geral e a mortalidade por neoplasia mamária, porém houve associaçáo significativa quando analisados os índices separadamente. Foi possível observar associação positiva do IDH renda e longevidade, e associação negativa do IDH educação.

A mulher com baixa renda per capita, baixa escolaridade, sem planos de saúde, sem companheiro, que reside em zona rural ou em área sem acesso para cuidados à saúde e a exames como o de mamografia, acaba possuindo menos chance de realizaçáo do rastreamento do câncer de mama com aumento da mortalidade $\mathrm{e}^{14,15}$.
Em estudo realizado por Sandovsky et al. ${ }^{15}$, sobre a relação do IDH e a prevenção do câncer de mama, houve um aumento de $6 \%$ da proporção de realização da mamografia a cada mudança de um desvio-padrão do IDH, encontrando, dessa forma, forte correlação positiva entre os dois. Dessa maneira, esses autores corroboram o presente estudo, uma vez que, particularmente entre as capitais com menor IDH, parece existir uma influência maior de fatores que dizem respeito à organização dos serviços de saúde e à acessibilidade aos métodos de prevenção e rastreamento do câncer de mama.

O fato de o presente estudo ter utilizado o Sistema de Informação de Mortalidade (SIM) pode significar limitação pelas diferenças regionais na cobertura, completude e qualidade da informaçấo. Atualmente, consideram-se de maior confiabilidade as informaçóes obtidas das Regióes Sul, Sudeste e Centro-Oeste do país, o que pode justificar, em partes, alguns padróes não esclarecidos de evolução temporal. Contudo, estudos anteriores apontam que os óbitos por neoplasia são os mais bem registrados, além disso, existe uma baixa cobertura dos Registros de Câncer de Base Populacional, tornando o SIM uma importante base para formulação de estratégias e políticas de saúde pública ${ }^{4,16}$.

\section{CONCLUSÃO}

Este estudo abre precedentes para estudos congêneres que possam abordar a tendência da mortalidade por câncer de mama de uma forma individualizada em cada município. Se faz necessário pensar em estratégias diferenciadas e fortalecimento de políticas nacionais que visem à prevençáo, à detecção precoce e ao acesso ao tratamento principalmente em cidades com altas taxas de mortalidade.

\section{CONTRIBUIÇÕES}

Larissa Di Leo Nogueira Costa contribuiu na concepçáo e planejamento do estudo, obtenção, análise e interpretaçáo dos dados e na redação do manuscrito. Rosangela Fernandes Lucena Batista contribuiu na concepçáo e planejamento do estudo, análise e interpretação dos dados, redação e revisão crítica do artigo. Maria Ieda Gomes Vanderlei, Ana Hélia de Lima Sardinha e Pabline Medeiros Verzaro contribuíram na obtenção e análise dos dados, redação e revisão crítica do artigo. Luciana Léda Carvalho Lisbôa contribuiu na redação e revisão crítica da versão final do artigo. Todas as autoras aprovaram a versão final do manuscrito.

\section{DECLARAÇÃO DE CONFLITO DE INTERESSES}

Nada a declarar. 


\section{FONTES DE FINANCIAMENTO}

Não há.

\section{REFERÊNCIAS}

1. Barbosa IR, Costa ICC, Pérez MMB, et al. Mortalidade por câncer de mama nos estados do Nordeste do Brasil: tendências atuais e projeções até 2030. Rev Ciência Plural. 2015;1(1):4-14.

2. Pinheiro AB, Lauter DS, Medeiros GC, et al. Câncer de mama em mulheres jovens: análise de 12.689 casos. Rev Bras Cancerol. 2013;59(3):351-359.

3. Instituto Nacional de Câncer José Alencar Gomes da Silva. A mulher e o câncer de mama no Brasil. 3. ed. rev. atual. Rio de Janeiro: INCA; 2018.

4. Girianelli VR, Gamarra CJ, Silva GA. Os grandes contrastes na mortalidade por câncer do colo uterino e de mama no Brasil. Rev Saúde Públ. 2014;48(3):459467. doi: https://dx.doi.org/10.1590/S00348910.2014048005214.

5. Toriola AT, Colditz GA. Trends in breast câncer incidence and mortality in the United States: implications for prevention. Breast Cancer Res Treat. 2013;138(3):665-673. doi: https://dx.doi.org/10.1007/s10549-013-2500-7.

6. Barbosa IR, Costa ICC, Pérez MMB, et al. As iniquidades sociais e as disparidades na mortalidade por câncer relativo ao gênero. Rev Ciência Plural. 2015;1(2):79-86.

7. Panis C, Kawasaki ACB, Pascotto CR, et al. Revisão crítica da mortalidade por câncer usando registros hospitalares e anos potenciais de vida perdidos. Einstein (São Paulo). 2018;16(1):1-7. doi: http://dx.doi. org/10.1590/s1679-45082018ao4018

8. Programa das Naçóes Unidas para o Desenvolvimento; Instituto de Pesquisa Econômica Aplicada; Fundação João Pinheiro. Atlas do desenvolvimento humano no Brasil [Internet]. Rio de Janeiro: PNUD; 2013 [acesso 2017 Mar 20]. Disponível em: http://www.atlasbrasil.org.br/2013/.

9. Instituto Brasileiro de Geografia e Estatística. Síntese de indicadores sociais: uma análise das condiçôes de vida da população brasileira: 2018. Rio de Janeiro: IBGE; 2018. (Estudos e pesquisas. Informação demográfica e socioeconômica; n. 39).

10. Soares LR, Gonzaga CMR, Branquinho LW, et al. Mortalidade por câncer de mama feminino no Brasil de acordo com a cor. Rev Bras Ginecol Obstet. 2015;37(8):388-92. doi: http://dx.doi.org/10.1590/ SO100-720320150005319.

11. Meira KC, Guimarães RM, Santos J, et al. Análise de efeito idade-período-coorte na mortalidade por câncer de mama no Brasil e regióes. Rev Panam Salud Públ. 2015;37(6):402-408.

12. Ministério da Saúde (BR); Secretaria de Vigilância em Saúde; Departamento de Vigilância de Doenças e Agravos não Transmissíveis e Promoção da Saúde. Vigitel Brasil 2017: vigilância de fatores de risco e proteção para doenças crônicas por inquérito telefônico: estimativas sobre frequência e distribuiçáo sociodemográfica de fatores de risco e proteção para doenças crônicas nas capitais dos 26 estados brasileiros e no Distrito Federal em 2017. Brasília: Ministério da Saúde; 2018.

13. Couto MSA. Análise da taxa de mortalidade por câncer de mama nos municípios brasileiros no período de 1987 a 2013 e fatores associados [dissertação]. Juiz de Fora, MG: Universidade Federal de Juiz de Fora; 2016.

14. Antoni S, Sasco AJ, dos Santos Silva I, et al. Is mammographic density differentially associated with breast cancer according to receptor status? A metaanalysis. Breast Cancer Res Treat. 2013;137(2):337-347. doi: https://doi.org/10.1007/s10549-012-2362-4.

15. Sadovsky ADI, Poton WL, Reis-Santos B, et al. Índice de desenvolvimento humano e prevenção secundária de câncer de mama e colo do útero: um estudo ecológico. Cad Saúde Públ. 2015;31(7):1539-50. doi: http://dx.doi. org/10.1590/0102-311X00073014.

16. Kubrusly LS. Utilização de técnicas de análise multivariada para redução de variáveis num problema de controle ecológico. Rev Bras Estat. 1992;53(199/200):53-67. 